\title{
Recruiting Subjects in Cancer Prevention and Control Studies
}

\author{
Mack T. Ruffin IV1* and John Baron² \\ ${ }^{1}$ D epartment of Family Medicine, U niversity of M ichigan M edical Center, Ann Arbor, M ichigan 48109 \\ 2D artmouth Medical School, Department of Community and Family M edicine, N orris Cotton Cancer \\ Center, Hanover, N ew Hampshire 03755-3861
}

\begin{abstract}
Recruitment of subjects is a critical aspect of prevention trials that is often overlooked by investigators. As a consequence, accrual time is often extended, workloads may become heavy, and resource utilization is increased in an effort to complete projects. Recruitment of healthy subjects in cancer prevention and control studies presents unique issues that need to be considered. The barriers to recruitment include participant issues, physician variables, features of the study design, and characteristics of the health care system in the U nited States. The authors provide their experience in overcoming these difficulties. J. Cell. Biochem. Suppl. 34:80-83, 2000. ๑ 2000 Wiley-Liss, Inc.
\end{abstract}

Key words: cancer prevention studies; controls; recruitment

The recruitment and retention of subjects are well-recognized hurdles for clinical trials [H unninghake et al., 1987; Lovato et al., 1997], and these difficulties demand focused efforts to overcome. Nonetheless, the scientific foundation to support these activities in prevention trials is embryonic, and what is known is largely based on treatment trials, not the healthy populations that are targets of most cancer prevention.

The intent of this article is to review the experience of the authors in recruiting and retaining subjects in clinical trials in identifying and overcoming barriers to recruitment and retention and to address issues unique to genetic and other high-risk cancer populations. A related area is the recruitment of minority populations; this issue that has been well reviewed elsewhere [Lovato et al., 1997; Swanson and Ward, 1995] and is not discussed here.

\section{BARRIERS TO RECRUITMENT}

In our experience, only a small percentage of the apparently eligible subjects will actually be randomized. Part of the problem is the frank

*Correspondence to: Mack T. Ruffin IV, Department of Family Medicine, University of Michigan Medical Center, 1018 Fuller Street, Ann Arbor, MI 48109-0708. E-mail: mruffin@umich.edu

Received 2 October 1998; Accepted 9 March 1999 ineligibility, which becomes apparent during the recruitment process. However, even among fully eligible subjects for prevention trials, only a modest proportion actually take part. In recent adenoma prevention trials, for example, less than $10 \%$ of subjects initially considered for inclusion were finally randomized. Successful recruitment of subjects into cancer prevention clinical trials involves a complex interaction between potential subjects and study staff, within the context of the specified study design. Therefore, barriers to recruitment can best be examined separately as issues arising from the participants' perspective, difficulties from the point of view of physicians and other health care providers, and problems emanating from the trial design itself.

\section{Participants' Perspective}

A distinguishing feature of prevention trials is the healthy character of the target population, in contrast to the relatively sick groups that characterize treatment trials. Since potential participants for prevention trials are well, and typically do not have medical problems that demand immediate attention, they may not feel that they will have much to gain by entering a trial. As a consequence, it is likely that subjects in a prevention trial are more highly selected than those in treatment trials. Characteristics of the participant play a major

(c) 2000 Wiley-Liss, Inc. 
role in this selection for recruitment. For example among women at high risk of breast cancer recruited to a clinical trial, education level was the key determinant of the impact of other characteristics in the decision to participate [Lerman et al., 1994].

Participant attitudes are also important determinants of recruitment. Participant attitudes and concerns have been classified into three general categories: feelings regarding time and effort involved in participation, personal and family attitudes regarding clinical trials, and perception of benefits from trial participation [Swanson and Ward, 1995]. Of these categories, it has been our experience that time and aggravation have the greatest influence on recruitment success. Concerns regarding waiting and travel time, time away from job and family, child care, and parking are among some of the issues. These may often be appropriateworries, but can easily be exaggerated. For example, the need to travel to an unfamiliar location may prompt concerns regarding such issues when in fact the perceived barriers do not really exist. Such problems are relatively easy to overcome by designing a system that is truly user-friendly and emphazing to the subjects the reality. This may include placing study sites in more convenient or familiar locations for thetargeted population.

Among some potential participants, there may bea fear of being a subject of investigation, in particular a hesitancy to submit to randomization. This concern may be more common among minority individuals, those who are less well educated, and those of lower socioeconomic class. Recruitment may also be difficult if evidence of the study's benefit (to the individual or to society) is uncl ear. This is an especially important oversight, as many participants enroll in prevention trials for al truistic reasons. Toavoid such problems, the authors have clarified the concerns of possible participants through community advisory boards, in-depth interviews with likely study participants, and focus groups.

\section{Physicians and 0 ther Health Care Providers}

The cooperation and participation of physicians and other health care providers are often essential to the success of recruitment to clinical trials; it will clearly be an important issuein multicentered studies. Physicians often serve as gatekeepers of access to trials; as such, they can either facilitate or prevent access to the trial. Indeed, the most common reason that a cancer patient is not enrolled in a trial is that the patient's physician made a decision not to enter that patient in the trial [F oley and Moertel, 1991].

Several factors contribute to whether physicians will be a barriers or facilitators. Trials requiring significant amount of time and effort for physicians or the office staff will not be welcomed. Studies interfering with the doctor/ patient relationship are also going to be rejected. This may be an ever-increasing barrier as patients are viewed as a commodity to physicians. Physicians may fear losing patients to competing clinicians if they are referred to participate in a trial. Finally, and most importantly, there is a conflict between physicians role as care giver and scientist. As caregivers, physicians place the interests of the individual first. As scientists, the benefit to humanity is placed first, including well-designed trials that use informed consent and randomization. Both issues are a dramatic paradigm shift from the daily activities of clinical practice.

The authors have used several strategies to overcome physician-related barriers. First, to the maximum extent possible, the trial can not add extra responsibilities to the workload of the physician or their office staff. This means that studies should have staff and resources to access the subjects, deliver the study protocol, and collect the data. Second, the single most important persons critical to success of any study in a physician's office are the front office staff such as the receptionist, tel ephone operator, and medical records staff. Thus, investigators should follow the lead of the drug company representatives and court these staff members with incentives and rewards-or at the very least treat them with a great deal of respect. Third, community-based physicians will greatly appreciate public acknowledgment and reward for their participation in studies. This goal can be accomplished with official appearing plaques for the office, local newspaper stories, or promotion of the physician as a local expert or resource at hospital staff meetings.

The health care system in the United States is also a factor that relates to the ability of physicians and other health care providers to recruit subjects. The health care system in the United States is not actually a system; rather, it can best be described as a chaotic, loosely connected network based primarily on employ- 
ment status. This makes it quite difficult to identify and access potential participants through a single, central source. One strategy to overcome this barrier is to use local organization that can serveas a single source of information. For example, managed care or health maintenance organizations can be a useful infrastructure to use to access subjects for cancer prevention and control trials [Hudmon et al., 1997].

\section{Trial D esign Barriers}

Trial designs and requirements are often very complex, and the complexity can be a significant barrier to recruitment. The intervention itself may be perceived as distasteful, such as diet changes or weight loss. The protocol may beinvasive, such as flexiblesigmoidoscopy with colorectal biopsies or even multiple blood draws. The intervention may be perceived as having too many undesirable side effects. Healthy subjects are not willing to take as much risk as are those who have illnesses. Thus, it is important that trials keep invasive procedures to a minimum, minimize the risk of adverse events, and maximize the possibility and evidence of benefit.

Perhaps the fundamental aspect of trial design that hampers completion of recruitment is simply the large sample sizes required. This typically forces a multicentered design that will magnify the administrative complexity of the study considerably. The fact that large numbers of subjects are required will put a premium on efficient and timely recruitment. In the Polyp Prevention Study Group, several facts about recruitment have become clear. First, clinicians regularly tend to overestimate the volume of suitable patients in their practices. Often, as clinicians consider their own practice, the eligibility criteria for of the trial being considered are discounted. For example, a clinician may perceive that their practice vol ume of colonoscopies is high. The perception of a large volume of eligible participants is false, since a substantial proportion of the practice consists of colon cancer follow-up, not colonic polyps. It is the volume of eligible subjects that is important. This has proven to be true of studies recruiting women undergoing col poscopy.

Second, once the planning is completed and recruitment actually begins, then it is important to introduce an active recruitment program. Relying on clinicians to remember to refer patients, or expecting advertisements and/or flyers to provide a brisk volume of patients, is unrealistic. Use of some sort of sampling frame is much more effective, such as a list of potentially eligible subjects who can be systematically approached to investigate participation. Other useful strategies are using recruitment coordinators, regular meetings (weekly or monthly) focused on recruitment, involvement of the principal investigator in recruiting (especially with interaction with other physicians), and setting goals. Finally, investigators should never fail to remind colleagues and reintroduce the enthusiasm for studies too often.

\section{Retention of Subjects}

Once the struggle to achieve a recruitment target is reached, there may be a natural tendency to breathe a sigh of relief and relax. Unfortunately, retention of randomized subjects, though typically less aggravating than recruitment, requires considerable sustained effort.

A run-period before randomization is one early strategy that can be employed to maintain the compliance of subjects in the trial. By identifying subjects who are likely to drop out, a run-in can provide some measure of protection. In the trials conducted by the Polyp Prevention Study Group, $15-20 \%$ of subjects who entered the run-in periods of the trials conducted have been deemed unsuitable for randomization. Presumably these subjects would have dropped out if they had been randomized.

Nonetheless, even subjects who successfully completetherun-in period may present difficulties regarding retention. In the relatively long trials of conducted by the Polyp Prevention Study Group (3-5 years), many of the dropouts have occurred because of comorbidity, and not directly because of the burdens of the study itself. This pattern is particularly difficult to combat-since no single characteristic of the trial has led to the dropout, there is not much specific that the study can do to prevent it, other than making continued participation as painless as possible. The approach has been to have study staff tailor a supportive approach to the individual subjects. For many subjects, this involves the establishment of a personal rapport entailing frequent contact regarding frustrations. This rapport is best established if study participants have continuity with the same re- 
search staff person throughout the duration of the study. Simple jesters such as birthdays and Holiday cards (used in the Polyp Prevention Study), inquiries about family members, listening to complaints about life stresses unrelated to the study, and assisting in solving unrelated health issues help to create committed study participants. From the point of view of both the clinicians and the study subjects, retention will be maximized if follow-up investigations are incorporated into routine care: following expected "routine" timing, and involving as little deviation from routine as possible.

\section{UNIQ UE ISSUES TO GENETIC AND HIGH-RISK IDENTIFICATIO N}

One unique aspect of some studies in cancer prevention and control is the identification of study subjects with genetic abnormalities. Difficult issues may arise both for the individual participant as well as for family members. Subjects participating in studies involving genetic testing have to consider risk such as discrimination and loss of insurability or employability if they are identified has carrying the genetic abnormality. In addition, family members of the study participants may face dealing with genetic information as well as discrimination similar to that the subject faces, but without informed consent. Not only are adults within the family affected, but children as well. These issues are only just beginning to be encountered among investigators and institutional review boards. There are currently no guidelines nor is clear that all of the risk are known.

\section{FUTURE PRO SPECTS}

Investigators need to place more effort on recruitment and retention of subjects in cancer prevention and control trials. This requires more understanding of the barriers and knowledge regarding the success of strategies to overcome these barriers. The published data on various strategies to improve recruitment and retention are very limited. More investigators are needed in this area. I nvestigators focusing more on the successes of their strategies and adding the experience to the published data would be extremely useful. The unique issues associate with participation in trials involving genetic testing needs more exploration.

\section{REFEREN CES}

F ol ey J F, Moertel CG. 1991. I mproving accrual into cancer clinical trials. J Cancer Educ 6:165-173.

Hudmon K, Honn S, J iang H, Chamberlain R, Xiang W, Ferry G, Gosbee W, Hong W, Spitz M. 1997. Identifying and recruiting healthy control subjects from a managed care organization: a methodol ogy for molecular epidemiological case-control studies of cancer. Cancer Epidemiol Biomarkers Prev 6:565-571.

Hunninghake DB, Darby CA, Probstfield J L. 1987. Recruitment experience in clinical trials: literature summary and annotated bibliography. Control Clin Trials 8:6S$30 \mathrm{~S}$.

Lerman C, Rimer B, Daly M, Lustbader E, Sands C, Balshem A, Masny A, Engstrom P. 1994. Recruiting highrisk women into a breast cancer health promotion trial. Cancer Epidemiol Biomarkers Prev 3:271-276.

Lovato LC, Hill K, Hertert S, Hunninghake DB, Probstfield J L. 1997. Recruitment for controlled clinical trials: Iiterature summary and annotated bibliography. Control Clin Trials 18:328-352.

Swanson GM, Ward AJ . 1995. Recruiting minorities into clinical trials: toward a participant-friendly system [see comments]. J Natl Cancer Inst 87:1747-1759. [Comment in: J Natl Cancer Inst 88:377.] 